\title{
Coupling between magnetic ordering and structural instabilities in perovskite biferroics: A first-principles study
}

\author{
Nirat Ray and Umesh V. Waghmare \\ Theoretical Sciences Unit \\ Jawaharlal Nehru Centre for Advanced Scientific Research \\ Jakkur PO, Bangalore 560 064, India
}

(Dated: November 13, 2018)

\begin{abstract}
We use first-principles density functional theory-based calculations to investigate structural instabilities in the high symmetry cubic perovskite structure of rare-earth $(\mathrm{R}=\mathrm{La}, \mathrm{Y}, \mathrm{Lu})$ and Bi-based biferroic chromites, focusing on $\Gamma$ and $R$ point phonons of states with para-, ferro-, and antiferromagnetic ordering. We find that (a) the structure with G-type antiferromagnetic ordering is most stable, (b) the most dominant structural instabilities in these oxides are the ones associated with rotations of oxygen octahedra, and (c) structural instabilities involving changes in $\mathrm{Cr}-\mathrm{O}-\mathrm{Cr}$ bond angle depend sensitively on the changes in magnetic ordering. The dependence of structural instabilities on magnetic ordering can be understood in terms of how super-exchange interactions depend on the $\mathrm{Cr}-\mathrm{O}-\mathrm{Cr}$ bond angles and $\mathrm{Cr}-\mathrm{O}$ bond lengths. We demonstrate how adequate buckling of $\mathrm{Cr}-\mathrm{O}-\mathrm{Cr}$ chains can favour ferromagnetism. Born effective charges (BEC) calculated using the Berry phase expression are found to be anomalously large for the A-cations, indicating their chemical relevance to ferroelectric distortions.
\end{abstract}

\section{INTRODUCTION}

A ferroic is a material which exhibits spontaneous and switchable ordering of electric polarization or magnetization or elastic strain. Materials exhibiting more than one of such orderings termed 'multiferroics'- have recently become the focus of much research ${ }^{1}$. Most of the biferroics investigated in recent years are $\mathrm{ABO}_{3}$ oxides with perovskite structure. The $d^{0}$-ness or the zero occupancy of tranistion metal $B$ cation is known chemically to favor ferroelectricity ${ }^{2}$. Hence, the availability of transition metal $d$-electrons in the perovskite oxides necessary for magnetism, reduces the tendency for off-centering ferroelectric distortions ${ }^{2}$ making multiferroics relatively rare. How the ordering of $d$-electronic spins of the $B$ cation influence ferroelectric or other competing structural instabilities has not yet been explored and understood. The coupling between magnetic ordering and structural instabilities is expected to involve interesting physics and is of direct relevance to technological applications ${ }^{\frac{3}{2}}$ such as multiple state memory elements and novel memory media.

There are at least three families of high temperature biferroic materials. Bi- based perovskite oxides,

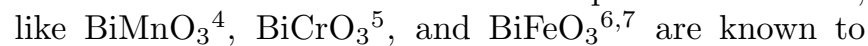
be promising biferroics. Ferroelectricity in these materials arises from the stereochemical activity of the $6 s$ lone pair electrons of $\mathrm{Bi}$. Hexagonal rare earth manganates $\mathrm{LnMnO}_{3}$ and $\mathrm{InMnO}_{3}$ are biferroics which exhibit improper or geometric ferroelectricity ${ }^{8,9,10,11}$. Rare earth chromites $\mathrm{LnCrO}_{3} 12,13$ (with $\mathrm{Ln}=\mathrm{Ho}, \mathrm{Er}, \mathrm{Tm}, \mathrm{Yb}, \mathrm{Lu}$ or $\mathrm{Y}$ ) have been recently shown to be biferroic; $\mathrm{YCrO}_{3}$ has been shown 12 to exhibit canted antiferromagnetic behavior below $140 \mathrm{~K}$ and a ferroelectric transition around $473 \mathrm{~K}$. Similarly, $\mathrm{LuCrO}_{3}$ becomes a canted antiferromagnet below $115 \mathrm{~K}$, and is ferroelectric below $488 \mathrm{~K}^{13}$. The absence of any ferroelctricity in $\mathrm{LaCrO}_{3}$, has been attributed to the large size of the $\mathrm{La}^{3+}$ ion, in comparison with $\mathrm{Y}^{3+}$.

However, small values of polarization reported for these materials (about $2 \mu \mathrm{C} / \mathrm{cm}^{2}$ for $\mathrm{YCrO}_{3} 12$ and $6 \mu \mathrm{C} / \mathrm{cm}^{2}$ of $\mathrm{BiFeO}_{3}{ }^{7}$ ) inspite of large A-cation offcentering distortions remain a puzzle. More recently $\stackrel{14}{ }$, a new concept of 'local non-centrosymmetry' in $\mathrm{YCrO}_{3}$ has been proposed to account for the small value of polarization observed. Perovskite oxides are known to have many competing structural instabilities $\frac{15}{}$ and this competition is further enriched by the magnetic instabilities. The coupling and competition between various magnetic and structural ordering can be partly responsible for weak ferroelectricity or the possibility of local non-centrosymmetry. Our goal here is to investigate this issue through determination of various structural instabilities for different magnetic orderings, with a focus on rare earth chromites.

We present results of detailed electronic structure and frozen phonon (at the $\Gamma$ and $\mathrm{R}$ points) calculations for a set of five materials $\left(\mathrm{LuCrO}_{3}, \mathrm{YCrO}_{3}, \mathrm{LaCrO}_{3}, \mathrm{BiCrO}_{3}\right.$ and $\mathrm{YFeO}_{3}$ ) in the cubic phase with three different magnetic orderings (para-, ferro- and antiferromagnetic). In Section II, we briefly describe the methods used in calculations here. In section III, we report results for structural energetics, the electronic density of states (DOS) and the Born Effective charges (BEC) for the cubic phase of these materials with different magnetic orderings. In Section IV, we report results for structural instabilities in chromites and compare them with those in a related compound $\mathrm{YFeO}_{3}$. Since the many-electron correlations are important in magnetic oxides, we estimate their effects on the structural instabilities through use of the Hubbard parameter $\mathrm{U}^{16}$. Using our results for structural instabilities, we show how certain nonpolar structural instabilities can cooperatively stabilize ferromagnetism. Our work reveals how the structural instabilities of these 
biferroic oxides depend on the size of A-cation (with the same B-cation), and a change in B-cation. We interpret the results using arguments based on superexchange 17 , and the well-known Goodenough-Kanamori rules ${ }^{18}$. Finally, we summarize in Section $\mathrm{V}$.

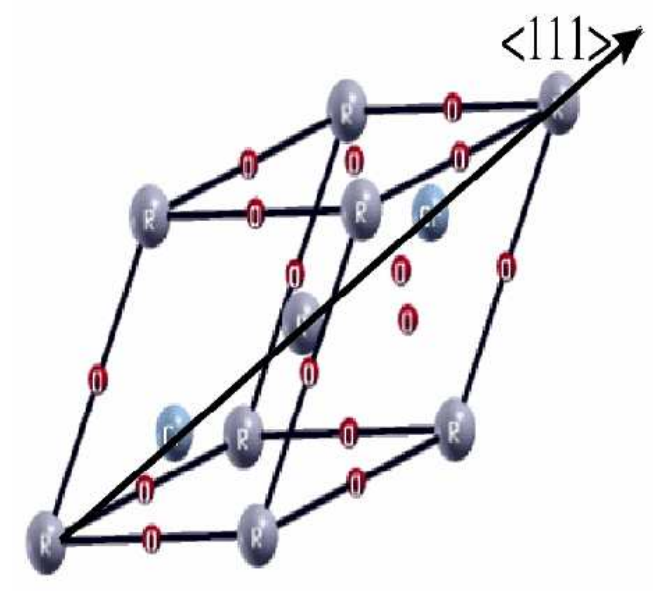

FIG. 1: Perovskite structure: Cell doubled along the $\langle 111\rangle$ direction, to represent G-type antiferroamgnetic ordering.

\section{METHODOLOGY}

Our calculations are based on first-principles pseudopotential based Density Functional Theory within a generalized gradient approximation (GGA)19 as implemented in the PWSCF package ${ }^{20}$. The interaction between ions and electrons is approximated with ultrasoft pseudopotentials 21 treating explicitly 11 electrons $\left[(n-1) s^{2}(n-1) p^{6}(n-1) d^{1}\right.$ and $\left.n s^{2}\right]$ in the valence shell of $\mathrm{Lu}(\mathrm{n}=6), \mathrm{La}(\mathrm{n}=6)$ and $\mathrm{Y}(\mathrm{n}=5)$. We consider 6 valence electrons for Oxygen $\left[2 \mathrm{~s}^{2} 2 \mathrm{p}^{4}\right]$ and 14 electrons for $\mathrm{Cr}$ $\left[3 \mathrm{~s}^{2} 3 \mathrm{p}^{6} 3 \mathrm{~d}^{5} 4 \mathrm{~s}^{1}\right]$. We used a plane wave basis with kinetic energy cut off of 25 Ryd (150 Ryd) to represent wave functions (density). For cubic structures, we sample the Brillouin zone using a $5 \times 5 \times 5$ Monkhorst Pack Mesh ${ }^{22}$, and a denser mesh $(6 \times 6 \times 6)$ and higher energy cut-off (30 Ryd) for energy differences. Phonon frequencies calculated with these larger parameters do not differ much from those calculated with a lower cut-off. We perform spin polarised calculations by initializing different spins on neighbouring magnetic ions; For paramagnetic ordering we initialise a zero spin on Cr ions. To represent antiferromagnetic ordering, the unit cell is doubled along the 〈111) direction (see Fig-1). To investigate structural instabilities in the prototype cubic structure, we determine its dynamical matrix using frozen phonon calculations. We use a finite difference form of the first derivative to compute an element of the force constant matrix:

$$
K_{i \alpha j \beta}=-\frac{\partial F_{i \alpha}}{\partial u_{j \beta}}
$$

TABLE I: Lattice constants of various oxides in the cubic perovskite structure with experimental unit cell volumes.

\begin{tabular}{lc|ccc}
\hline \hline & & \multicolumn{3}{c}{ Stress(GPa) } \\
\hline & $\mathrm{a}(\AA)$ & PM & FM & AFM \\
\hline $\mathrm{LuCrO}_{3}$ & 3.77 & -4.712 & 3.302 & 1.429 \\
$\mathrm{YCrO}_{3}$ & 3.79 & -3.875 & 3.883 & 2.240 \\
$\mathrm{LaCrO}_{3}$ & 3.88 & -7.043 & -0.083 & -1.564 \\
$\mathrm{YFeO}_{3}$ & $3.83^{a}$ & -15.8 & -5.9 & 2.5 \\
$\mathrm{BiCrO}_{3}$ & $3.85^{b}$ & -15.4 & -9.7 & -11.2 \\
\hline \hline
\end{tabular}

${ }^{a} \operatorname{Ref} 25$

${ }^{b} \operatorname{Ref} \underline{\underline{4}}$.

$$
=-\frac{F_{i \alpha}\left(u_{j \beta}=\Delta\right)-F_{i \alpha}\left(u_{j \beta}=-\Delta\right)}{2 \Delta}
$$

where $\mathrm{F}_{i \alpha}$ is the Hellman-Feynman force acting on the $i^{\text {th }}$ atom in $\alpha$ direction, and, $\mathrm{u}_{j \beta}$ the displacement of the $j^{\text {th }}$ atom in $\beta$ direction with respect to the equilibrium structure. We used $\Delta=0.04 \stackrel{\circ}{A}$, about $1 \%$ of the lattice constant. The dynamical matrix is then calculated from the force constant matrix,

$$
D_{i \alpha j \beta}=\frac{K_{i \alpha j \beta}}{\sqrt{m_{i} m_{j}}},
$$

whose eigenvalues correspond to the square of the phonon frequencies $\left(\omega^{2}\right)$.

In periodic systems, the dynamical charge tensor or Born effective charge tensor can be defined ${ }^{23}$ as the coefficient of proportionality between the macroscopic polarization created in direction $\beta$ and a rigid displacement of the sublattice of atoms $j$ in direction $\alpha$,

$$
Z *_{j, \alpha \beta}=\Omega_{o} \frac{\partial P_{\beta}^{e l}}{\partial u_{j, \alpha}},
$$

$\Omega_{o}$ being the unit cell volume. The polarization is determined using the berry phase formalism ${ }^{24}$ as implemented in the PWSCF package.

\section{PROPERTIES OF THE CUBIC PEROVSKITE STRUCTURE}

We have determined electronic structure of $\mathrm{RCrO}_{3}$ compounds $(\mathrm{R}=\mathrm{Y}, \mathrm{Lu}, \mathrm{La})$ in the high symmetry cubic structure with different magnetic orderings. This is accomplished through calculations with different initial guesses for atomic spin polarization and optimizing with respect to spin density. All our calculations are for the experimental unit cell volumes, as ferroelectricity is known to be sensitive to lattice constants or pressure (see lattice constants listed in Table \). In many magnetic compounds, a change of magnetic ordering causes a stress which induces a structural distortion ${ }^{26}$. This concept of 'magnetic stress' was introduced to describe structural phase transitions that are induced by magnetic ordering, and applied to materials with degenerate (usual $\mathrm{e}_{g}$ ) 
$\operatorname{orbitals}^{27}$. In these materials as well, a change in magnetic ordering (with fixed lattice parameters) produces a change in stress (see Table \). For $\mathrm{LuCrO}_{3}$ and $\mathrm{YCrO}_{3}$, the introduction of spin polarization produces a change from compressive stress in the paramagnetic phase to a tensile stress, with stress being minimum in the antiferromagnetic structure (the lowest energy ordering). In $\mathrm{LaCrO}_{3}$, the stress remains compressive with all three magnetic orderings.

\section{A. Electronic structure of $\mathrm{YCrO}_{3}, \mathrm{LuCrO}_{3}$ and $\mathrm{LaCrO}_{3}$}

\section{Cubic Paramagnetic(PM) Structure}

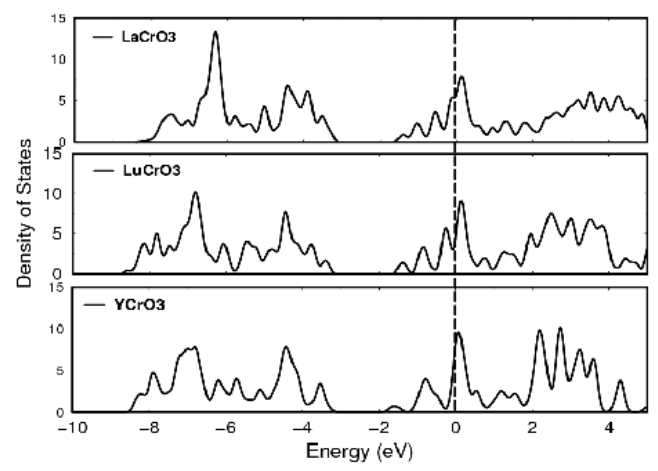

FIG. 2: Density of States for cubic PM YCrO $3, \mathrm{LuCrO}_{3}$ and $\mathrm{LaCrO}_{3}$. The fermi level (indicated by a dashed line) has been set to zero in all the three cases.

First, we present results for the highest symmetry cubic structure with no spin polarization. Although this state is experimentally inaccessible, it provides a useful reference for understanding the spin-polarized structures discussed later in the paper. The plotted energy range is from -10 to $4 \mathrm{eV}$, and the lower lying semicore states have been omitted for clarity. In the $\mathrm{PM}$ cubic $\mathrm{YCrO}_{3}$, $\mathrm{LuCrO}_{3}$ and $\mathrm{LaCrO}_{3}$, (see Fig 2) there is high density of electronic states at the Fermi level, driving the system towards a Stoner instability 28 . This suggests that this phase should be unstable with respect to spin polarization and/or structural distortions. The contribution of various orbitals to the DOS can be understood better by examining the orbital projected density of states (see Fig 3) which show that, the contribution between -8 to $-3 \mathrm{eV}$ is mainly from Oxygen $2 p$ orbitals. Cr d-orbitals contribute predominantly to the peaks at the Fermi level. In contrast, the contribution from the $\mathrm{Lu}$ d-orbitals is substantial only $2 \mathrm{eV}$ above the Fermi level.

\section{Cubic Ferromagnetic(FM) Structure}

Ferromagnetic cubic structure is simulated by initializing spins on both $\mathrm{Cr}$ ions in the same direction. In

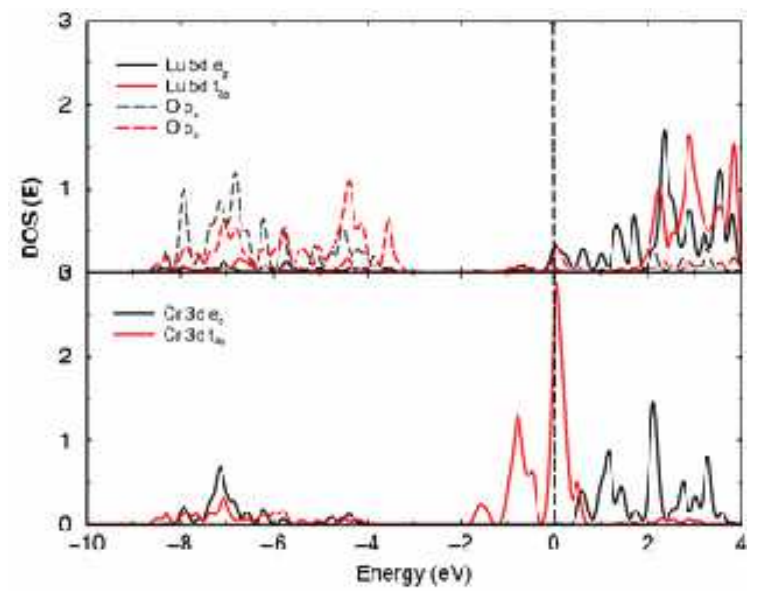

FIG. 3: Orbital resolved density of states for cubic PM $\mathrm{LuCrO}_{3}$. The high density of states at the Fermi level hints that it is an unstable phase.
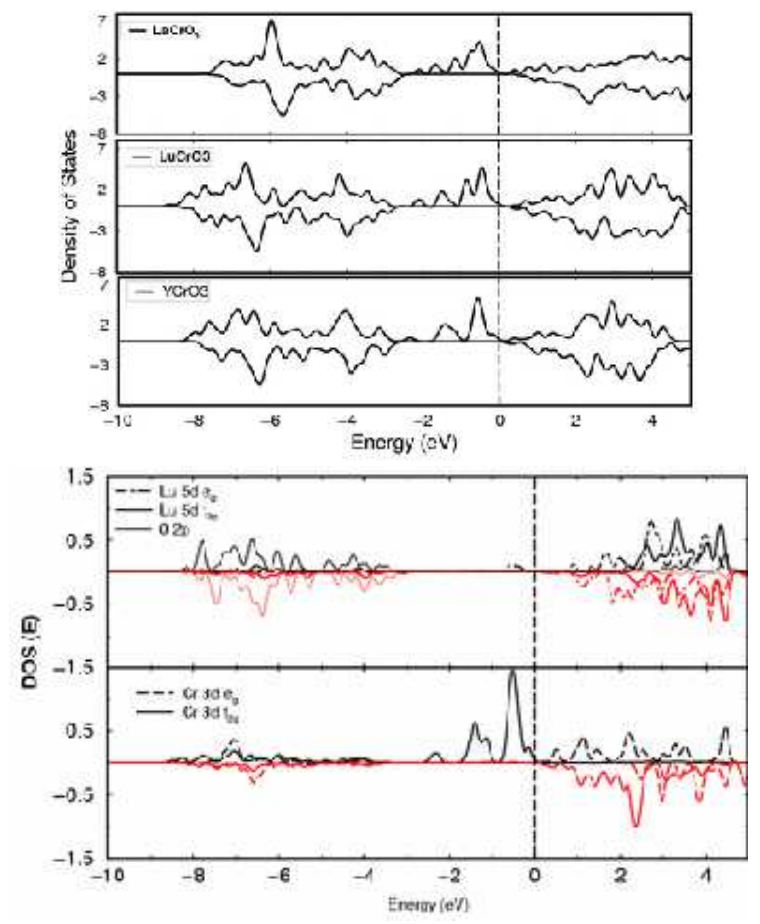

FIG. 4: Total and orbital resolved Density of states for cubic ferromagnetic $\mathrm{YCrO}_{3}, \mathrm{LuCrO}_{3}$ and $\mathrm{LaCrO}_{3}$ with the Fermi level set to zero in all the three cases.

all chromites studied here, the ferromagnetic structures have a magnetic moment of $3 \mu_{B}$ in accordance with the Hund's rule value expected for a $\mathrm{d}^{3}$ configuration. The majority spins are represented by the solid line on the positive $\mathrm{Y}$ axis, and the minority spins on the negative $\mathrm{Y}$ axis. The introduction of spin polarization reduces the energy by approximately $2 \mathrm{eV}$ per unit cell. The source of stabilization is clear from the density of states (see Fig 4) which reveals opening of a gap at the Fermi level. The states corresponding to non-magnetic atoms 
are unchanged in comparison with PM ordering. The down-spin $\mathrm{Cr} 3 d$ states are split off from the $\mathrm{O} 2 p$ states creating a wide gap for the minority states. The up-spin $\mathrm{Cr} 3 d$ states hybridize with the $\mathrm{O} 2 p$ states and there is a very small gap for the majority carriers. The density of electronic states at the Fermi level is still finite having a small contribution from the $\mathrm{Cr}$ d-orbitals. This hints that either the ferromagnetic phase may not be the most stable, and that either an antiferromagnetic (AFM) spin arrangement could lower the energy of the system, or that the cubic structure is unstable and a structural distortion will lower the energy of the system. Since $\mathrm{Cr}^{3+}$ is a $d^{3}$ ion, it is Jahn-Teller inactive, and the structural distortions (if any) probably involve the A-cation (at the corners), or the oxygen anions.

\section{Antiferromagnetic Structure}

We simulated antiferromagnetic structure by initializing antiparallel spins on the two Cr ions in the supercell. It is well known that, the superexchange between $\mathrm{e}_{g}$ orbitals of adjacent ions connected through oxygen with a $180^{\circ}$ metal-oxygen-metal bond angle, is much stronger than the interaction between the corresponding $\mathrm{t}_{2 g}$ orbitals, since the former is mediated by stronger $\operatorname{dp} \sigma$ bonds as compared to the weaker $\operatorname{dp} \pi$ bonds in the latter ${ }^{17,18}$. So, we expect a superexchange interaction in which there is a weak coupling between the $\mathrm{t}_{2 g}$ orbitals of the adjacent $\mathrm{Cr}$ atoms giving rise to an antiferromagnetic interaction. Further, this coupling will be stronger in the cubic structure as the bond angle between $\mathrm{Cr}-\mathrm{O}-\mathrm{Cr}$ is $180^{\circ}$, as compared to a distorted structure. From su-

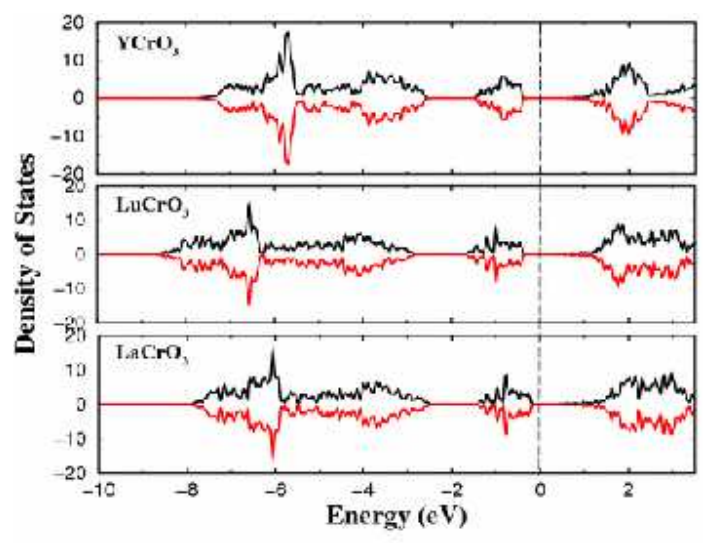

FIG. 5: Density of states for antiferromagnetic $\mathrm{YCrO}_{3}$, $\mathrm{LuCrO}_{3}$ and $\mathrm{LaCrO}_{3}$.

perexchange arguments applied to $d^{3}$ configurations, the structure with G-type antiferromagnetic ordering having rhombohedral symmetry should be most stable. We consider collinear spins assuming that the canting of the spins would be small. We find that the AFM structure is lower in energy by about $0.4 \mathrm{eV}$ than the FM phase. We note that this gain in energy by FM ordering with respect to $\mathrm{PM}$ ordering is much more (around $2 \mathrm{eV}$ ) than the gain in energy in going from the $\mathrm{FM}$ to the $\mathrm{AFM}$ structure (see Table III). $\mathrm{LuCrO}_{3}$, like $\mathrm{YCrO}_{3}$, is also found to be insulating with the introduction of a gap at the Fermi level (see Fig. 5). Both spin channels have identical density of states consistent with an AFM spin arrangement.

From the orbital projected density of states for AFM arrangement, we find that the $\mathrm{t}_{2 g}$ orbitals of $\mathrm{Cr}$ are fully occupied (and constitute the HOMO), whereas the $\mathrm{e}_{g}$ orbitals are unoccupied. Although the LUMO consists of $\mathrm{Cr}$ d-orbitals (about $2 \mathrm{eV}$ above the Fermi level), the $\mathrm{Lu} d$ states also appear within the same energy range (see Fig. 6).

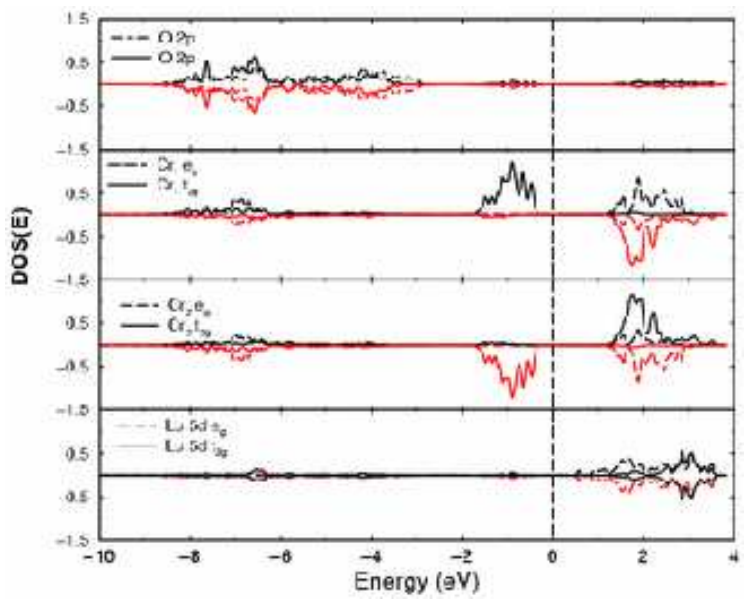

FIG. 6: Orbital resolved density of states for antiferromagnetic $\mathrm{LuCrO}_{3}$.

\section{B. Born Effective Charge (BEC)}

The effective charge tensors have been calculated from polarization differences between the perfect and distorted structures in the AFM phase. The anomalous values of $\mathrm{Z}^{*}$ so obtained indicate that a large force is felt by a given ion due to small macroscopic electric field, thus favoring a tendency for off-centering and toward a polarized ground state. The effective charge of the A-cation (see Table III) is about the same for the three cases. For $\mathrm{LaCrO}_{3}$, we find a larger $\mathrm{BEC}$ on $\mathrm{Cr}$ and one of the oxygen atom moving along the bond. This is possibly because of larger Cr-O bond length (arising from larger size of La cation, see Table III) and correspondingly greater contribution from the long-range charge transfer. We expect from this, that the superexchange interaction in $\mathrm{LaCrO}_{3}$ should be stronger as well. 
TABLE II: The XX component of Born effective charge tensor for AFM LaCrO $3, \mathrm{LuCrO}_{3}$ and $\mathrm{YCrO}_{3}$. Nominal charges are indicated in brackets.

\begin{tabular}{lcccc}
\hline \hline & $\mathrm{Z}^{*}$ & $\mathrm{Z}{ }_{B}$ & $\mathrm{Z} * O_{x}$ & $\mathrm{Z}^{*} O_{y, z}$ \\
\hline $\mathrm{LuCrO}_{3}$ & $4.42(3)$ & $3.43(3)$ & $-2.56(-2)$ & $-2.62(-2))$ \\
$\mathrm{YCrO}_{3}$ & $4.45(3)$ & $3.44(3)$ & $-2.62(-2)$ & $-2.66(-2))$ \\
$\mathrm{LaCrO}_{3}$ & $4.5(3)$ & $3.76(3)$ & $-3.82(-2)$ & $-2.22(-2))$ \\
\hline \hline
\end{tabular}

TABLE III: Relative Energies of different Magnetic phases, Cr-O bond lengths, and Neel's temperatures for cubic $\mathrm{LuCrO}_{3}, \mathrm{YCrO}_{3}$ and $\mathrm{LaCrO}_{3}$ (Energy of the PM phase has been set to zero).

\begin{tabular}{cccccc}
\hline \hline & PM & FM & G-AFM & Cr-O Bond length & $T_{N}$ \\
\hline $\mathrm{LuCrO}_{3}$ & 0.0 & $1.89 \mathrm{eV}$ & $2.3 \mathrm{eV}$ & $1.88(\AA)$ & $115 \mathrm{~K}^{a}$ \\
$\mathrm{YCrO}_{3}$ & 0.0 & $2.02 \mathrm{eV}$ & $2.4 \mathrm{eV}$ & $1.90(\AA)$ & $140 \mathrm{~K}^{a}$ \\
$\mathrm{LaCrO}_{3}$ & 0.0 & $2.04 \mathrm{eV}$ & $2.5 \mathrm{eV}$ & $1.94(\AA)$ & $282 \mathrm{~K}^{b}$ \\
\hline \hline
\end{tabular}

${ }^{a}$ Neel's temperature taken from Ref. 12 13.

${ }^{b}$ Ref. 4.

\section{STRUCTURAL INSTABILITIES}

\section{A. Coupling with Magnetic Ordering}

In order to represent G-type antiferromagnetic ordering which has been shown to be most favourable energetically, we use a unit cell doubled along the $\langle 111\rangle$ direction. We determine structural instabilities in this structure with different magnetic orderings. A single unit cell has 10 atoms which results in 30 phonon branches: 3 accoustic (which have zero frequency at $\mathrm{k}=(0,0,0)$ ) and 27 optical, some of which are triply degenerate. We are interested mainly in optical modes with imaginary phonon frequencies corresponding to instabilities in the structure. Doubling the unit cell along the $\langle 111\rangle$ direction, gives us access to zone boundary phonon modes (R-point) which form the dominant structural instabilities in this structure, along with the zone-center modes.

In the paramagnetic phase, both $\mathrm{YCrO}_{3}$ and $\mathrm{LuCrO}_{3}$ exhibit a zone center instability at 116.5 and $144.8 \mathrm{~cm}^{-1}$ respectively, which is a polar mode (with $\Gamma_{15}$ symmetry) involving mainly the off centering of A-cation. This instability, however, is absent in $\mathrm{LaCrO}_{3}$. We find two instabilities in the FM phase: $\Gamma_{15}$ and $\Gamma_{25}$ modes. The non-polar $\Gamma_{25}$ mode involves oxygen displacements only, and is strongly unstable in the FM phase. The $\Gamma_{15}$ mode involves the A-cation (rare earth ion) moving in a direction opposite to that of the oxygen cage and $\mathrm{Cr}$ atom resulting in a ferroelectric polar structural distortion. Note that the $\mathrm{Cr}$ atom moves in the same direction as the oxygen ion, in contrast to the behavior of $\mathrm{Ti}$ ion in $\mathrm{BaTiO}_{3} 15$ and $\mathrm{PbTiO}_{3} 29$, but similar to the behavior of $\mathrm{Mn}$ in $\mathrm{BiMnO}_{3}{ }^{11}$ and $\mathrm{Cr}$ in $\mathrm{BiCrO}_{3}{ }^{4}$.

With AFM spin arrangement, for $\mathrm{LuCrO}_{3}$, we find three triply degenerate instabilities, at $339.5 \mathrm{~cm}^{-1}, 145$ $\mathrm{cm}^{-1}$ and the weakest at around $60 \mathrm{~cm}^{-1}$. For $\mathrm{YCrO}_{3}$, the corresponding instabilities are at $309 \mathrm{~cm}^{-1}, 140 \mathrm{~cm}^{-1}$ and $80 \mathrm{~cm}^{-1}$ respectively. The strongest instability (at around $300 \mathrm{~cm}^{-1}$ for the two materials) has $\mathrm{R}_{25}$ symmetry and corresponds to rotation of the corner connected oxygen octahedra. The next instability is the ferroelectric $\Gamma_{15}$ mode (around $140 \mathrm{~cm}^{-1}$ ). The weakest instability $\left(60 \mathrm{~cm}^{-1}\right.$ for $\mathrm{LuCrO}_{3}$ and $80 \mathrm{~cm}^{-1}$ for $\left.\mathrm{YCrO}_{3}\right)$ has $\mathrm{R}_{15}$ symmetry and involves displacement of the $\mathrm{A}$ cations (Lu and Y for our case) and small oxygen displacements; these are antiparallel in neighbouring unit cells. In $\mathrm{LaCrO}_{3}$, we find only two triply degenerate instabilities. The first instability at around $220 \mathrm{~cm}^{-1}$ corresponding to the oxygen rotations (the $R_{25}$ mode) and the second close to $18 \mathrm{~cm}^{-1}$ having $\Gamma_{15}$ symmetry (see Fig (7).
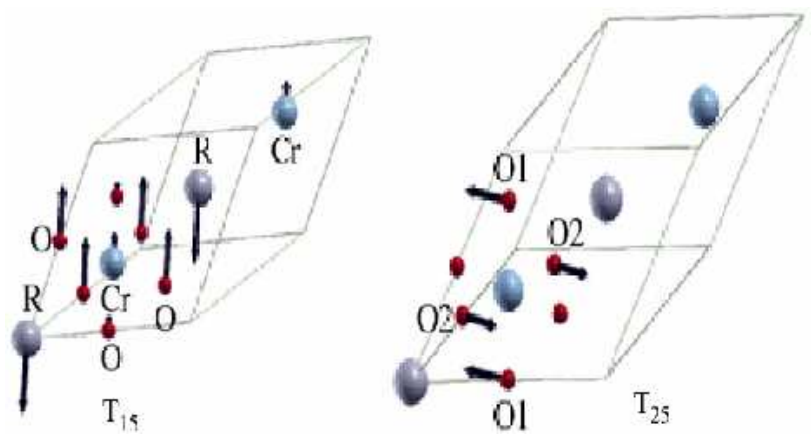

FIG. 7: Eigenvectors of the unstable $\Gamma$ point phonon modes: $\Gamma_{15}$ and $\Gamma_{25}$ modes

For the rare earth chromites studied in this paper, unstable R-point modes in the AFM phase do not change significantly with magnetic ordering. $\Gamma$ point instabilities in contrast depend strongly on magnetic ordering. Only the high frequency phonons are affected in going from para- to antiferromagnetic phase and all $\Gamma_{15}$ and $R_{15}$ modes are softer in the FM phase.

The $\Gamma_{25}$ mode which brings about a significant change in the $\mathrm{Cr}-\mathrm{O}-\mathrm{Cr}$ as well as $\mathrm{O}-\mathrm{Cr}-\mathrm{O}$ bond angle shows a spectacular change with magnetic ordering. This mode, highly unstable in the ferromagnetic phase (at around $200 \mathrm{~cm}^{-1}$ ) becomes stable at around $50 \mathrm{~cm}^{-1}$ with para- and antiferromagnetic orderings. This behavior, although not as pronounced, is also seen for this mode in $\mathrm{LaCrO}_{3}$. The $\mathrm{R}_{25}$ instability, also involving a change in the $\mathrm{Cr}-\mathrm{O}-\mathrm{Cr}$ bond angle, is not affected by the change in magnetic ordering possibly because the O-Cr-O bond angle still remains unchanged.

Another significant change is observed for the $R_{25}^{\prime}$ mode, which involves a movement of the two B-cations ( $\mathrm{Cr}$ in our case) in opposite directions. After introduction of spin-polarization, the $\mathrm{R}_{25}$ ' mode (close to $250 \mathrm{~cm}^{-1}$ for PM phase) becomes more stable at around $400 \mathrm{~cm}^{-1}$ for the FM and AFM phases.

We compare our results with the Bi-based biferroic chromite, $\mathrm{BiCrO}_{3}$ and find a similar behavior of the $\Gamma_{25}$ 
mode here as well. We thus attribute this behavior to the $\mathrm{B}$ cation ( $\mathrm{Cr}$ ion for the chromites) and expect it to be their general behavior. To interpret the general trend in phonon frequencies (see Fig. 8), the following rules apply:

1. The modes which involve a change in the $\mathrm{Cr}-\mathrm{O}-$ $\mathrm{Cr}$ bond angle (as well as $\mathrm{O}-\mathrm{Cr}-\mathrm{O}$ bond angle) are more stable in the AFM phases and are relatively less, or unstable, in the FM phase. This behaviour is seen in the $\Gamma_{25}$ and $\Gamma_{15}$ modes for $\mathrm{LuCrO}_{3}$.

2. Secondly, the modes which involve a change in the Cr-O bond length tend to harden with the introduction of spin polarization, as observed for the $\mathrm{R}_{25}^{\prime}$ and $\mathrm{R}_{12}$ modes.

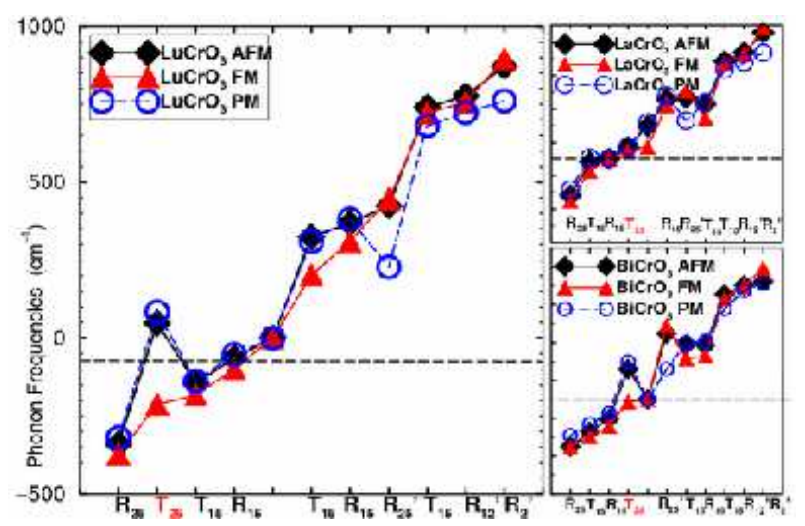

FIG. 8: Changes in phonon frequencies of cubic $\mathrm{LuCrO}_{3}$ with different magnetic orderings. Insets show similar curves for $\mathrm{LaCrO}_{3}$ and $\mathrm{BiCrO}_{3}$.

In order to study the effect of change in B-cation (see Fig 9), we compare instabilities in $\mathrm{YFeO}_{3}$ with $\mathrm{YCrO}_{3}$. We find that a change in magnetic ordering has an opposite effect on the unstable modes involving a change in Fe-O-Fe bond angle. A spectacular change is the $\mathrm{R}_{2}$ mode, an oxygen breathing mode, which softens in the FM phase as compared to the PM and AFM phases. The $\Gamma_{25}$ mode also shows a different behaviour, showing a stabilization with FM ordering. These differences are due to the filled $\mathrm{e}_{g}$ orbitals in $\mathrm{Fe}^{3+}$, which are unoccupied in $\mathrm{Cr}^{3+}$, leading to a much stronger superexchange interaction mediated by the $\mathrm{e}_{g}$ orbitals, and have a different geometry dependence.

\section{B. Stabilization of Ferromagnetic Ordering}

The spectacular change in the frequency of the $\Gamma_{25}$ mode with FM ordering, prompts us to discuss whether ferromagnetism can be stabilized in these chromites by varying the $\mathrm{Cr}-\mathrm{O}-\mathrm{Cr}$ bond angle. Since the $\Gamma_{25}$ mode is unstable only in the FM phase, we want to study the effect of freezing in a distortion of this mode. We have calculated the total energy as a function of $\Gamma_{25}$ displacements in the $\langle 111\rangle$ and $\langle 100\rangle$ directions, for the FM and AFM phases (see Fig 10). For $\mathrm{LuCrO}_{3}$, we observe a

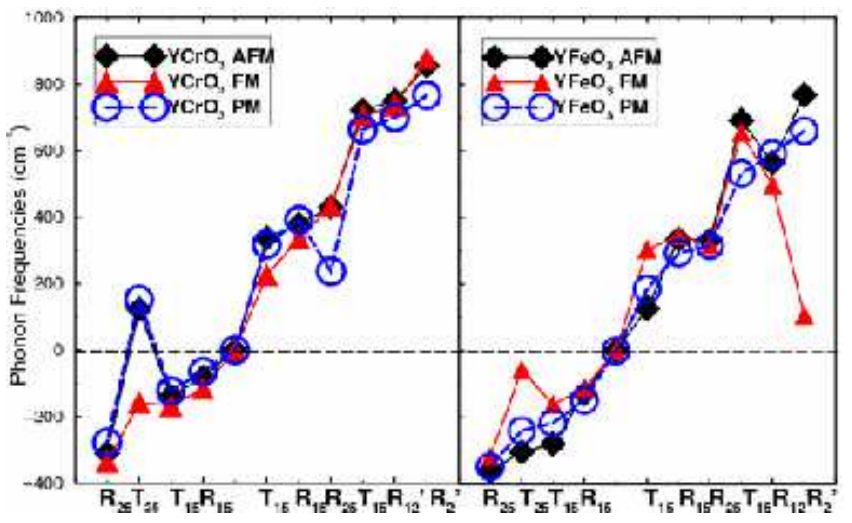

FIG. 9: Changes in phonon frequencies with changes in Bcation: $\mathrm{YCrO}_{3}$ and $\mathrm{YFeO}_{3}$.

crossover, at a displacement of $0.407 \stackrel{\circ}{A}$, beyond which the FM phase is energetically favoured. The $\mathrm{Cr}-\mathrm{O}-\mathrm{Cr}$ bond angle, at the crossover point is found to be $153^{\circ}$, which is significantly different from, the value suggested by Goodenough and Kanamori for ferromagnetic superexchange interaction $\left(130^{\circ}\right)$ in 1951 . On examining the Density of states beyond the crossover point (see Fig. 11), we find more significant hybridization between $\mathrm{Cr}$ and oxygens for the FM phase. Secondly, the FM phase so stabilized is found to be insulating.

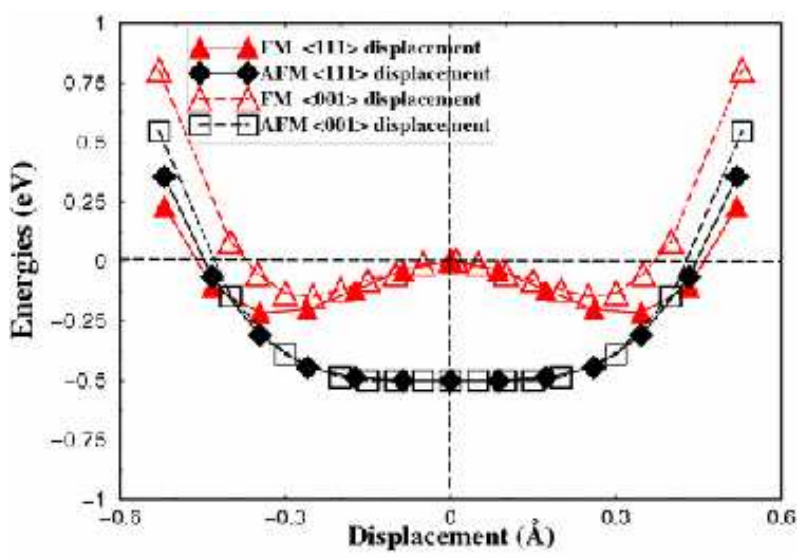

FIG. 10: Energy vs displacement corresponding to the $\Gamma_{25}$ mode for FM and AFM orderings in $\mathrm{LuCrO}_{3}$. FM becomes more stable than the AFM state for rhombohedral distortions greater than $0.407 \stackrel{\circ}{A}$.

\section{Effect of Correlations}

As mentioned earlier in the paper, the LDA+U method has been successfully applied to describe the electronic structure of sytems containing localized $d$ and $f$ electrons where LDA sometimes leads to incorrect results ${ }^{16}$, and recently it has been applied to obtain structural parameters that are in better agreement with experimental 


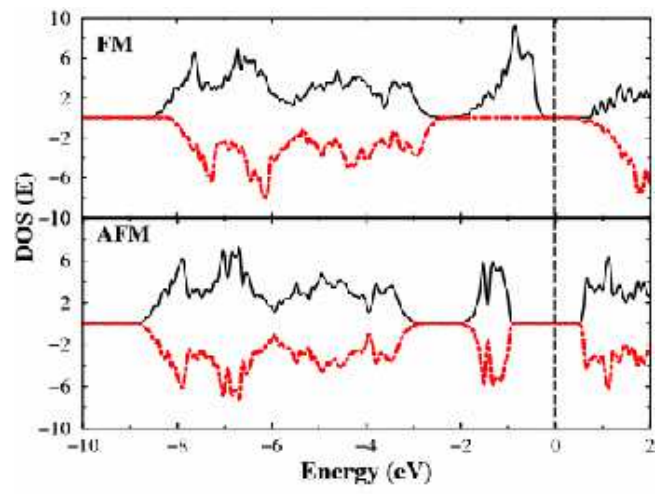

FIG. 11: Density of states for FM and AFM phases at $\Gamma_{25}$ displacement of $0.407 \AA$ (see Fig. 10, where the FM state is more stable).

results than LDA or GGA.7.30. In this work, we use a value of $\mathrm{U}=3.0 \mathrm{eV}$, adapted from work on full structural optimization of $\mathrm{BiCrO}_{3}{ }^{7}$. With the introduction of correlations through $\mathrm{U}$ parameter, we find that the modes in FM phase do not change significantly. Our results for $\mathrm{YCrO}_{3}$ (see Fig (12) bear that only $\Gamma_{25}, \mathrm{R}_{2}^{\prime}$ and $\mathrm{R}^{\prime}{ }_{12}$ modes are noticeably affected. Correlations lead to softening of the $\mathrm{R}_{2}^{\prime}$ and $\mathrm{R}_{12}^{\prime}$ modes by $50 \mathrm{~cm}^{-1}$, and tend to harden the $\Gamma_{25}$ mode in the PM phase. In an AFM spin arrangement, these R-point modes are hardened by approximately $20 \mathrm{~cm}^{-1}$ and the $\Gamma_{25}$ instability is softened.

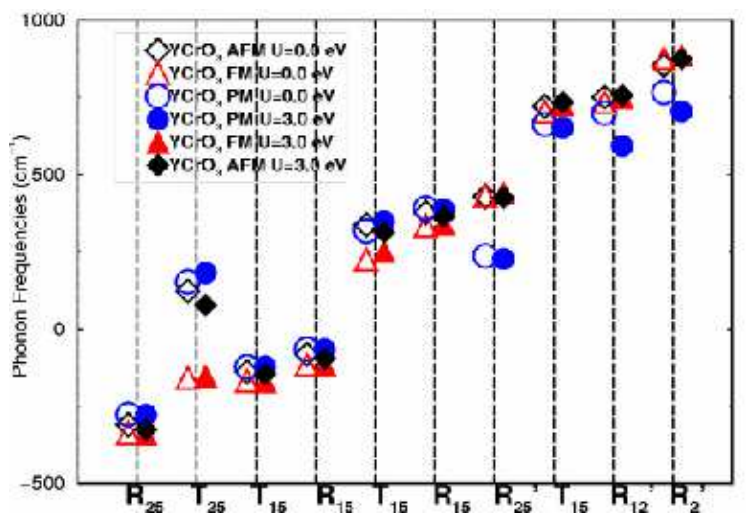

FIG. 12: Effect of correlations on the phonon modes of $\mathrm{YCrO}_{3}$ with different magnetic orderings. For each mode, data on the left of the vertical dashed line represents estimates with GGA and the data on the right represents estimates with $G G A+U$ $(\mathrm{U}=3.0 \mathrm{eV})$.

\section{SUMMARY}

In conclusion, we have determined structural instabilities of $\mathrm{LaCrO}_{3}, \mathrm{LuCrO}_{3}, \mathrm{YCrO}_{3}$ and $\mathrm{BiCrO}_{3}$ in their cubic perovskite structures with different magnetic orderings. Our finding that the G-type antiferromagnetic ordering is most stable can be explained with superexchange arguments. Ferroelectric structural instabilities in the cubic structures involve A-cation ( $\mathrm{Lu}$ or $\mathrm{Y}$ ) displacements, as indicated by the eigenvectors of the ferroelectric $\Gamma_{15}$ modes and an anomalous BEC of the Acation. We find that certain phonon frequencies depend sensitively on magnetic ordering: the modes involving a change in bond-angle are stable (harder) with the antiferro- and paramagnetic ordering than in the FM state; on the other hand, the modes involving a change in $\mathrm{Cr}-\mathrm{O}$ bond length are softer in the paramagnetic phase and comparable in the FM and AFM states. The $\Gamma_{25}$ oxygen mode brings about a significant change in the $\mathrm{Cr}$ O-Cr bond angle and is highly unstable in the FM phase, and corresponding structural distortion leads to stabilization of ferromagnetic ordering in these chromites. Among the competing structural instabilities the antiferrodistortive instability ( $R_{25}$ mode) is the strongest. Electron correlations are found to have little effect on the unstable phonon modes, but result in a slight change in a few of the stable phonon modes in the PM phase. We note that the effects of magnetic ordering on structural instabilities are quite different (in fact, opposite sometimes) in $\mathrm{YFeO}_{3}$ with respect to chromites, and a more detailed study is required to understand such couplings in ferrites. Origin of small polarization and/or local non-centrosymmetry 14 is probably from the relatively weak ferroelectric instabilities and their competition with various structural magnetic instabilities, and our work should be useful in formulating a phenomenological analysis of the same.

\section{ACKNOWLEDGMENTS}

Nirat Ray thanks JNCASR for Summer Research fellowship Programme and Joydeep Bhattacharjee for discussions. UVW is thankful to Professor C N R Rao for stimulating discussions and encouragement for this work and acknowledges use of central computing facility and financial support from the Centre for Computational Materials Science at JNCASR.
1 N. A. Spaldin, Phys. World, April 2004; T. Kimura et al Nature 426, 55 (2003); N. A. Spaldin and M. Fiebig, Science 309, 391 (2005).

2 N.A. Hill, J. Phys. Chem. B 104, 6684-6709 (2000).

3 Wood, V. E.; Austin, A. E. Magnetoelectric Interaction
Phenomena in Crystals; Freeman, A. J., Schmid, H., Eds.; Gordon and Breach: Langhorne, PA, 1975.

4 N.A. Hill and K.M. Rabe, Phys. Rev. B 59, 8759 (1999); A. Moreira dos Santos et al, Phys. Rev. B 66, 064425 (2002).

5 N.A. Hill, Pio Bättig and Claude Daul, J. Phy Chem B 
106, 3383-3388 (2002).

6 J. Wang et al., Science 299, (5613), 1719 (2003).

7 J. B. Neaton et al, Phys. Rev. B 71, 014113 (2005).

8 B. B. VanAken, Thomas T.M. Palstra1, A. Filippetti and N. A. Spaldin, Nat. Mat. 3, 164 (2004).

9 C. R. Serrao et al., J. Appl. Phys. 100, 076104 (2006).

10 C. J. Fennie and K. M. Rabe, Phys. Rev. B 72, 100103(R) (2005).

11 M. Feibig, J. Magn. Magn. Mater. 290-291, 883 (2005).

12 C. R. Serrao et al., Phys Rev B 72, 220101 (2005).

13 J. R. Sahu et al., J. Mater. Chem. 17, 42-44 (2007).

14 K. Ramesha et al., J. Phys. Cond. Matter 19, 102202 doi:10.1088/0953-8984/19/10/102202

15 Ph Ghosez, E. Cockayne, U.V. Waghmare and K.M. Rabe, Phys. Rev. B 60, 836 (1999).

16 V. I. Anisimov, F. Aryasetiawan and A. I. Lichtenstein, J. Phys. Cond. Matter 9, 767 (1997).

17 P. W. Anderson, Phys. Rev. 79, 350 - 356 (1950).

18 J. B. Goodenough, Phys. Rev. 100564 (1955).

19 Y. Wang and J. P. Perdew, Phys. Rev. B 44, 13298 (1991).

20 S. Baroni, A. Dal Corso, S. de Gironcoli, and P. Giannozzi, 2001 , http://www.pwscf.org

21 D. Vanderbilt, Phys. Rev. B 41, 7892 (1990).

22 H.J. Monkhorst and J.D. Pack, Phys. Rev. B 13, 5188 (1976); 16, 1748 (1977).

23 Ph. Ghosez, J. P. Michenaud, and X. Gonze, Phys. Rev. B 58, 6224 (1998).

${ }^{24}$ R. Resta, Berry Phase in Electronic Wave functions, available at: http://ale2ts.ts.infm.it:6163/ resta/publ/notes_trois.ps.gz

25 M. Eibschitz, Acta. Cryst. 19, 337 (1965).

26 Alessio Filippetti and Nicola A. Hill, Phys. Rev. Lett. 85, 5166 - 5169 (2000).

27 P. Gopal, N. A. Spaldin and U. V. Waghmare, Phys. Rev. B 70, 205104 (2004).

28 E.C. Stoner, Proc. R. Soc. 165, 372 (1938).

29 R. E. Cohen, Nature 358, 136138 (1992).

30 O. Bengone, M. Alouani, J. Hugel, and P. Blöchl, Comput. Mater. Sci. 24, 192 (2002).

31 A. Kokalji, XCrySDen - a new program for displaying crystalline structures and electron densities, J. Mol. Graphics Modelling 17, 176 (1999). 